\title{
El conocimiento especializado de un profesor de matemáticas: Un estudio de caso sobre la enseñanza de los conceptos básicos de función
}

\author{
Mathematics teachers' Specialized Knowledge: A Case Study about Teaching of the \\ Basic Concepts of Function
}

O conhecimento especializado de um professor de matemática: Um estudo de caso sobre o ensino dos conceitos básicos de função

\author{
Ariana Rodríguez-Flores \\ arodriguez89@gmail.com \\ Universidad Nacional \\ Heredia, Costa Rica \\ Miguel Picado-Alfaro \\ miguepicado@hotmail.com \\ Universidad de Costa Rica \\ San José, Costa Rica \\ Jonathan Espinoza-González \\ espinozaj25@gmail.com \\ Universidad Nacional \\ Heredia, Costa Rica \\ Nielka Rojas-González \\ nielka001@gmail.com \\ Universidad Católica del Norte \\ Antofagasta, Chile
}

Recibido-Received: 26/feb /2017 / Corregido-Corrected: 20/mar /2017.

Aceptado-Accepted: 2/may /2017 / Publicado-Published: 31/ene /2018.

\begin{abstract}
Resumen
Esta investigación enfoca el conocimiento especializado que muestra un profesor de matemática de secundaria al enseñar los conceptos básicos de función. Se enmarca en las investigaciones sobre el conocimiento matemático del profesor para la enseñanza (Hill, Ball y Schilling, 2008). Además, emplea el análisis didáctico (Gómez, 2007) para determinar indicadores que permitan identificar y caracterizar este conocimiento. Los resultados muestran la riqueza de la estructura conceptual presentada durante la enseñanza, así como un uso preciso del lenguaje matemático por parte del docente y la utilización de diversos sistemas de representación, enfatizando la relación entre ellas.

Palabras claves: Docente de secundaria; enseñanza de las matemáticas; formación de docentes de secundaria; matemáticas.
\end{abstract}


UNICIENCIA Vol. 32, No. 1, pp. 89-107. Enero-Junio, 2018.

ISSN Electrónico: 2215-3470

URL: www.revistas.una.ac.cr/uniciencia

DOI: http://dx.doi.org/10.15359/ru.32-1.6

Email: revistauniciencia@una.cr

\begin{abstract}
This research draws its attention to the specialized knowledge a secondary school teacher of mathematics demonstrates while teaching the basic concepts of function. This paper falls within the research field of teachers' mathematical knowledge for teaching (Hill, Ball and Schilling, 2008). In addition, it uses didactic analysis (Gómez, 2007) to determine indicators that allow the identification and the characterization of this knowledge. The results show the richness of the conceptual structure taught when teaching, as well as the precise use of the mathematical language by the teacher, and the use of diverse systems of representation that emphasize the relation between them.
\end{abstract}

Keywords: Secondary teachers; Mathematics teaching; Secondary teachers training; mathematics.

\begin{abstract}
Resumo
Esta pesquisa enfoca o conhecimento especializado que um professor de matemática da secundária mostra ensinando os conceitos básicos de função. Enquadra-se nas pesquisas sobre o conhecimento matemático do professor para o ensino (Hill, Ball e Schilling, 2008). Além disso, emprega a análise didática (Gómez, 2007) para determinar indicadores que permitam identificar e caracterizar este conhecimento. Os resultados mostram a riqueza da estrutura conceptual apresentada durante o ensino, assim como um uso preciso da linguagem matemática por parte do docente e a utilização de diversos sistemas de representação, enfatizando a relação entre elas.
\end{abstract}

Palavras-chaves: Docente de secundária; ensino da matemática; formação de docentes de secundária; matemática.

El profesor o profesora es un agente crucial en los procesos de enseñanza y aprendizaje de la matemática. Entre sus funciones está la de guiar al estudiantado en la construcción de su conocimiento, con todo lo que esto conlleva, pero principalmente conocer en profundidad el saber a enseñar antes de ser presentado (Espinoza, Espinoza, González, Ramírez y Zumbado, 2008).

Diversas investigaciones sobre el conocimiento matemático resaltan la importancia de que el personal docente debe poseer una buena preparación matemática para ser más efectivo al momento de enseñar la materia. Por ejemplo, Haciomeroglu (2006) indica que docentes con un fuerte conocimiento matemático tienen mejor preparación para ayudar a sus estudiantes a comprender significativamente el tema y que quien estimule a sus grupos con preguntas y les brinde diferentes puntos de vista sobre el tema, evidencia gran conocimiento matemático y didáctico sobre este.

En Costa Rica, en el año 2010, el Ministerio de Educación Pública realizó una prueba a docentes de matemática de educación secundaria del país para evaluar su conocimiento matemático. Los ítems evaluados eran similares a los que se presentan en el examen de bachillerato (prueba estandarizada que realizan estudiantes de quinto año de secundaria para obtener el título de Bachillerato en Educación Secundaria). Los resultados indican que 43,3\% de docentes no domina completamente parte de los contenidos que se estudian en la educación secundaria (Cuarto Informe Estado de la Educación, 2013). Esto evidencia la falta de un conocimiento especializado en algunos de los contenidos que se evaluaron en la prueba, lo cual podría influir en la enseñanza de dichos conceptos y en el rendimiento académico del estudiantado. A esto es importante agregar que el Ministerio de Educación Pública no tiene un mecanismo para asegurar que el personal docente que contrata posea estos conocimientos más allá del título profesional obtenido en carreras, que difieren hasta en dos años de tiempo en el mismo grado profesional 
ISSN Electrónico: 2215-3470

DOI: http://dx.doi.org/10.15359/ru.32-1.6
UNICIENCIA Vol. 32, No. 1, pp. 89-107. Enero-Junio, 2018. URL: www.revistas.una.ac.cr/uniciencia Email: revistauniciencia@una.cr

(Ruíz, Barrantes y Gamboa, 2009; Morales-López, 2017). En el caso de estudiantes, los resultados de las pruebas de Bachillerato en Matemática muestran la dificultad que tienen en esta materia (MEP, 2012a). En general, muchos de los contenidos presentan niveles de aprobación menor al 50\%, particularmente el tema de funciones es uno de los que presenta un bajo porcentaje de aprobación. No obstante, esta dificultad en el manejo de contenidos relacionados con las funciones se presenta también tanto en el profesorado de matemática en ejercicio como en quienes están realizando su formación inicial (Argawal, 2006; Morales y Font, 2017; Shumway, 2003).

El concepto de función es esencial para el estudiantado, puesto que permite describir las relaciones de cambio entre las variables, explicar los cambios de parámetros e interpretar y analizar gráficos (Clement, 2001). Por lo tanto, no sorprende que los principios y estándares para las matemáticas escolares (NCTM, 2000) y las nuevas políticas educativas costarricenses (MEP, 2012b) promuevan la introducción de conceptos vinculados a patrones, relaciones y funciones a partir de edades más tempranas.

Dada la importancia del tema de funciones, así como de la dificultad en su aprendizaje y al débil manejo de los contenidos relacionados con el tema por una parte de docentes, es de interés estudiar el conocimiento matemático del profesorado de matemática en el tema de funciones.

En este sentido, el presente estudio se enmarca en las investigaciones sobre el conocimiento matemático del profesorado para la enseñanza, iniciadas por Shulman (1986, 1987) y desarrolladas por Ball y colaboradores (Ball, Hill y Bass, 2005; Ball, Thames y Phelps, 2008; Hill, Ball y Schilling, 2008). Este modelo enfatiza la diferencia entre el conocimiento del contenido matemático (CCM) y el conocimiento pedagógico del contenido (CPC). Además, propone tres subdominios para cada uno de estos conocimientos. También se empleó el análisis didáctico (Gómez, 2007) y los aportes de Rojas, Flores y Ramos (2013) para identificar dominios de conocimiento matemático específicos ligados al concepto matemático abordado.

Los resultados que se presentarán corresponden al dominio del CCM, enfocados en el subdominio del conocimiento especializado del contenido (CEC) (ver otros resultados sobre este dominio en Rodríguez-Flores, Picado-Alfaro, Espinoza-González, Rojas-González y Flores-Martínez, 2016). Así, el problema de investigación abordado es caracterizar el CEC de un profesor de matemática al enseñar los conceptos básicos de función, en cuarto año de la educación secundaria en Costa Rica (estudiantes con edades entre 15 y 17 años). Para lograrlo se han definido tres objetivos específicos:

1. Describir el proceso de enseñanza que sigue un profesor de matemática al enseñar los conceptos básicos de función.

2. Identificar los componentes del CEC que manifiesta un profesor de matemática al enseñar los conceptos básicos de función.

3. Determinar indicadores sobre el CEC que caracterizan a un profesor de matemática que enseña los conceptos básicos de función.

Este trabajo pretende aportar información sobrela importancia del conocimiento matemático que debería tener el personal docente, principalmente para la toma de decisiones de parte de las autoridades educativas del país y los entes encargados de formar al futuro profesorado, al permitir fortalecer la formación de estos grupos profesionales en la enseñanza de la matemática en aquellos temas con mayor dificultad para el estudiantado. 


\section{Marco teórico}

La fundamentación teórica de la investigación toma base el modelo teórico sobre el conocimiento matemático para la enseñanza y el análisis didáctico. A continuación, se detallan algunas ideas de estas propuestas.

\section{Conocimiento profesional del profesorado de matemática}

El conocimiento profesional del profesorado corresponde a aquellos saberes y experiencias que pone en práctica el personal docente, que viene construyendo desde su formación y durante el desarrollo de su profesión (Climent, 2002).

En sus estudios, Shulman $(1986,1987)$ ha precisado las diferencias entre el conocimiento del contenido y el conocimiento del contenido para la enseñanza. El primero corresponde a la cantidad y a la organización del contenido en la mente del profesorado (Shulman, 1986). En este sentido el personal docente debe ser capaz de manejar las definiciones y poder justificar proposiciones en particular, así como conocer las relaciones del conocimiento con otras disciplinas.

Por otra parte, el conocimiento del contenido para la enseñanza se refiere a la combinación del contenido y la pedagogía (Shulman, 1987). El profesorado debe analizar la forma de presentar la materia a sus estudiantes, tomando en cuenta las habilidades y dificultades que puedan presentarse, además de adaptarla a la diversidad de interés del grupo de jóvenes.

\section{Dominios del conocimiento matemático para la enseñanza (CME)}

Hill, Ball y Schilling (2008) definen el conocimiento matemático para la enseñanza como "el conocimiento matemático que los profesores utilizan en el aula para producir aprendizaje y crecimiento en los alumnos" (p. 374). Así, el profesorado debe poseer un conocimiento específico para la enseñanza que va más allá del conocimiento matemático.

A partir de los planteamientos de Shulman (1986, 1987), Ball, Thames y Phelps (2008).proponen un modelo del conocimiento matemático para la enseñanza en el que enfatizan la diferencia entre dos dominios de conocimiento: Conocimiento del contenido matemático y conocimiento pedagógico del contenido matemático. Asimismo, establecen tres subdominios para cada uno de los dominios propuestos. En la figura 1 se muestra el modelo propuesto por los autores.

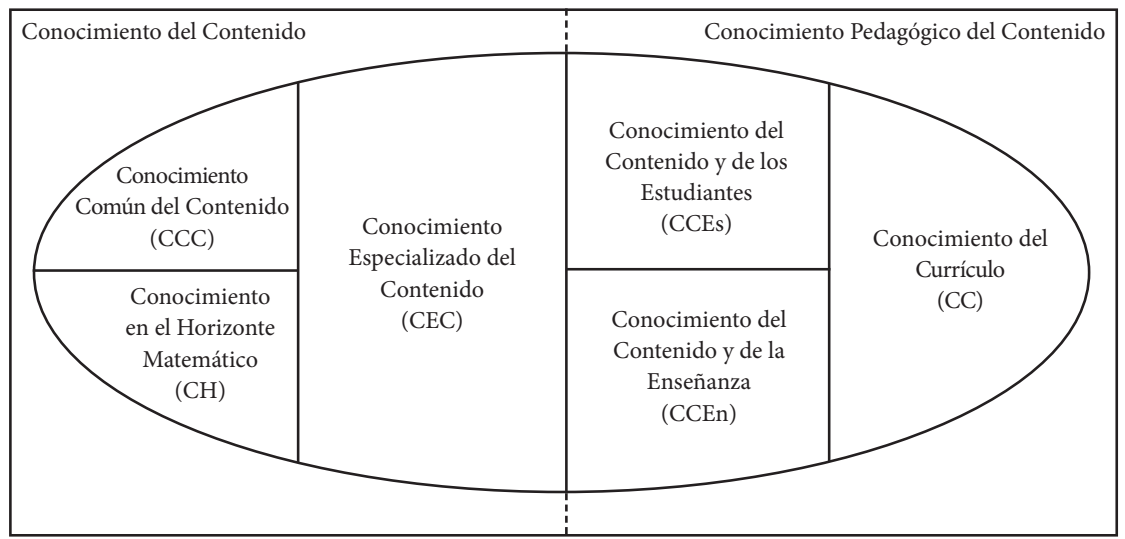

Figura 1. Dominios de conocimiento matemático para la enseñanza (Ball, Thames y Phelps, 2008). Extraído de Content Knowledge for Teaching: What Makes It Special? Journal of Teacher Education, 59(5), 389-407. 
ISSN Electrónico: 2215-3470 DOI: http://dx.doi.org/10.15359/ru.32-1.6
UNICIENCIA Vol. 32, No. 1, pp. 89-107. Enero-Junio, 2018. URL: www.revistas.una.ac.cr/uniciencia Email: revistauniciencia@una.cr

\section{Subdominios del conocimiento del contenido matemático}

El conocimientodel contenido matemático incluye el conocimiento común del contenido(CCC), el conocimiento especializado del contenido (CEC) y el conocimiento del horizonte matemático $(\mathrm{CH})$. Para este estudio solo se considerará el CEC, el cual corresponde al conocimiento que le permite al profesorado realizar tareas relacionadas con la enseñanza, como brindar definiciones, argumentaciones y definiciones matemáticas precisas y adecuadas (Ball, Hill y Bass, 2005).

Por ejemplo, para organizar una secuencia de enseñanza con la cual lograr el aprendizaje de diferentes aspectos de un contenido determinado, el profesor tiene que tener un conocimiento que va más allá del conocimiento matemático que se aprende en la escuela, lo cual exige del docente poseer un conocimiento matemático y competencias específicas. (Rojas, 2010, p. 11)

Por tanto, esta investigación se enfoca en el conocimiento matemático que muestra un docente al enseñar los conceptos básicos de función en el cuarto año de la educación secundaria costarricense, principalmente destaca la amplitud y precisión del lenguaje matemático empleado, la diversidad de sistemas de representación utilizados y sus relaciones, la variedad de significados que presenta de un mismo concepto, el grado en que las situaciones que emplea dan sentido al contenido matemático escolar, la relación de las tareas empleadas con los significados y su adecuación a las situaciones y contextos, la riqueza y claridad de las respuestas a las preguntas y dudas de sus estudiantes, entre otros.

En relación con el conocimiento pedagógico del contenido (CPC), que acentúa los procesos de enseñanza y el aprendizaje de las matemáticas, se compone por tres subdominios: Conocimiento del contenido y del estudiantado, conocimiento del contenido y de la enseñanza y conocimiento del currículo. Estos dominios no son considerados en este estudio. Para una mayor caracterización de estos pueden consultarse los trabajos de Hill, Ball y Schilling (2008) y Rojas (2010).

\section{Análisis didáctico}

Otro referente teórico usado en este estudio es el análisis didáctico, "procedimiento con el que es posible explorar, profundizar y trabajar con los diferentes y múltiples significados del contenido matemático escolar, para efectos de diseñar, llevar a la práctica y evaluar actividades de enseñanza y aprendizaje" (Gómez, 2007, pp. 18-19). Este está compuesto por cinco análisis parciales: análisis conceptual, de contenido, cognitivo, de instrucción y de evaluación.

El análisis conceptual explora la diversidad de significados de un concepto matemático, concretamente el análisis de contenido destaca la estructura conceptual, la fenomenología y sistemas de representación utilizados que se vinculan con estos conceptos matemáticos (Rojas, 2010). Este análisis se realiza, por ejemplo, mediante la revisión de libros de texto. Además, el personal docente debe disponer de una organización del contenido para elegir las tareas que realizará en el proceso de enseñanza (Rojas, Flores y Ramos, 2013).

El análisis cognitivo se estructura en expectativas, limitaciones y oportunidades en el aprendizaje escolar (Lupiáñez, 2009). Las expectativas son los fines, objetivos y capacidades, para establecer caminos de aprendizaje. Las limitaciones se refieren a los errores y dificultades que tiene el estudiantado al aprender un tema. Las oportunidades muestran las tareas planteadas o propuestas para la enseñanza de un contenido (Rojas, Flores y Ramos, 2013). 
En el análisis de instrucción el profesorado diseña, analiza y selecciona una variedad de tareas como elementos de la unidad didáctica de planificación. Analiza también instrumentos de evaluación y recursos didácticos (Lupiáñez, 2009).

El análisis de evaluación logra describir las habilidades y dificultades que ha manifestado el estudiantado durante el proceso escolar (Gómez, 2007). Este permite una evaluación de los análisis previos y su utilidad para establecer modificaciones e iniciar nuevamente el ciclo de análisis.

Para identificar el conocimiento que pone en práctica el profesor para enseñar los conceptos básicos de función, se emplea la propuesta desarrollada Rojas, Flores y Ramos (2013), en la que se relacionan los componentes del análisis didáctico con el conocimiento matemático para la enseñanza. Para establecer estas relaciones se consideran tres análisis parciales: de contenido, cognitivo e instrucción y las distintas dimensiones que lo conforman.

De esta forma, el personal docente manifiesta un conocimiento especializado de los conceptos básicos de función (SCK, por sus siglas en inglés) cuando, además de resolver de forma correcta tareas relacionadas a este contenido, enseña los conceptos básicos de función empleando distintos significados, utiliza diversidad de representaciones (gráficas, simbólicas, entre otras), destaca el sentido de los conceptos mediante el estudio de las situaciones y contextos que muestra su utilidad y aplicabilidad (fenomenología de los conceptos); también, al mostrar relaciones entre los elementos de la estructura conceptual de las funciones, por ejemplo, entre las tareas de aprendizaje con la diversidad de significados.

\section{Metodología}

El estudio corresponde a una investigación cualitativa basada en el estudio de casos (McMillan y Schumacher, 2005). Describe y caracteriza el conocimiento especializado del contenido que manifiesta un profesor de matemáticas al enseñar los conceptos básicos de función a un grupo de estudiantes de cuarto año de educación secundaria, de un colegio de modalidad académica diurna en Costa Rica.

\section{Descripción del participante}

Este artículo expone la información proporcionada por este profesor de matemáticas de educación secundaria. La selección del profesor se orientó por criterios que respondían al objetivo del estudio, los planteamientos del equipo investigador del proyecto en que se enmarca esta investigación y la propuesta para la selección de docentes con experticia realizada por Rojas, Carrillo y Flores (2012).

Los criterios de selección tomaron en cuenta los siguientes aspectos: (a) interés y disponibilidad del profesor para participar en la investigación; (b) trabajar en una institución pública de educación secundaria de la provincia de Heredia; (c) disponibilidad para que sus clases sean observadas y grabadas en video; (d) disponibilidad para contestar algún instrumento de ser necesario, (e) ser profesor propietario, esto por si es necesario durante el proceso de investigación volver a recolectar algún dato; $y$, (f) tener al menos tres años de experiencia en la enseñanza de las funciones. También, se consideraron otras características para seleccionar docentes con experticia, como tener una experiencia mínima de cinco años en la enseñanza de las matemáticas y ser consciente del cambio curricular en matemáticas. 


\section{Recolección de la información}

Los datos se recolectaron mediante la observación no participante de lecciones de matemáticas (denominadas episodios). Estos episodios fueron registrados en audio y video. Para tal fin se solicitó la autorización correspondiente a las autoridades de la institución, al profesor, a los padres y madres de familia y a estudiantes; se garantizó el anonimato durante las intervenciones de clase. Para realizar un proceso sistemático de recolección de información se elaboró un protocolo de observación a partir de las categorías y de las unidades de análisis consideradas.

\section{Categorías y proceso de análisis}

El análisis se llevó a cabo a partir de la adaptación de las categorías propuestas por Rojas, Flores y Ramos (2013) para el estudio del conocimiento especializado del profesorado de matemáticas. El establecimiento de las unidades de análisis requirió el análisis didáctico de las funciones desde los principios del análisis de contenido (estructura conceptual, fenomenología y sistemas de representación) descrito en el marco teórico. La tabla 1 muestra las categorías y las unidades de análisis consideradas.

Tabla 1

Categorías y unidades de análisis para el CEC

Categorías Unidades de análisis

Riqueza de las relaciones entre elementos de la Las explicaciones del profesor muestran riqueza de relación estructura conceptual (EC) entre conceptos (pueden aparecer otros conceptos). Los ejemplos, ejercicios y problemas que utiliza el profesor muestran riqueza de relación entre conceptos. Introduce conceptos básicos de función a partir de relaciones entre conceptos matemáticos. Utiliza la relación entre los conceptos para responder a las preguntas de sus estudiantes.

Variedad de contextos que aparecen en las tareas planteadas $(\mathrm{VC})$
Utiliza contextos de interés para sus estudiantes. Aprovecha contextos de aplicación. Emplea contextos sobre historia de la matemática. Utiliza contextos de problemática nacional. Emplea contextos que reflejen algún eje transversal (especificar en observaciones).

Plantea situaciones para mostrar el uso del concepto de: cantidad constante; cantidad variable; variable dependiente; variable independiente; relación; función; dominio; codominio; ámbito o rango; imagen; preimagen; criterio de una función; gráfica de una función; otros conceptos.

Presenta un significado del concepto y varios significados.

Variedad de significados del concepto que pone en juego y grado en que se explicitan (VS)

Diversidad de sistemas de representación del concepto y frecuencia con la que la utiliza (DR)
Concepto: cantidad constante; cantidad variable; variable dependiente; variable independiente; relación; función; dominio; codominio; ámbito o rango; imagen; preimagen; criterio de una función; gráfica de una función; otros conceptos. Sistema de representación: verbal, icónica, tabular, gráfica, simbólica algebraica, simbólica numérica, otras.

continúa.. 


\begin{tabular}{ll}
\hline \multicolumn{1}{c}{ Categorías } & \multicolumn{1}{c}{ Unidades de análisis } \\
\hline $\begin{array}{l}\text { Realce que concede a cada uno de los sistemas } \\
\text { de representación utilizado (RSR) }\end{array}$ & $\begin{array}{l}\text { Presenta más de cinco veces este tipo de representación. Presenta } \\
\text { los beneficios de su uso. Utiliza la representación para mostrar } \\
\text { más de un concepto. }\end{array}$ \\
\hline $\begin{array}{l}\text { Amplitud y precisión del lenguaje matemático } \\
\text { (LM) }\end{array}$ & $\begin{array}{l}\text { Muestra riqueza del lenguaje matemático formal en la } \\
\text { presentación de conceptos. Utiliza con fluidez el lenguaje } \\
\text { matemático formal para la presentación de los conceptos. El } \\
\text { lenguaje matemático formal utilizado para el desarrollo de los } \\
\text { conceptos es preciso. }\end{array}$ \\
\hline $\begin{array}{l}\text { Establecimiento de relaciones entre } \\
\text { representaciones (RER) }\end{array}$ & $\begin{array}{l}\text { Sistema de representación: verbal, icónica, tabular, gráfica, } \\
\text { simbólica algebraica, simbólica numérica, otras. }\end{array}$ \\
\hline $\begin{array}{l}\text { Riqueza y claridad de respuestas a las } \\
\text { preguntas y dudas de sus estudiantes (RRE) }\end{array}$ & $\begin{array}{l}\text { Muestra disposición para atender las preguntas y dudas } \\
\text { de sus estudiantes. Atiende con variedad de respuestas las } \\
\text { preguntas y dudas de sus estudiantes. Expresa con claridad } \\
\text { las respuestas a las preguntas y dudas de sus estudiantes. }\end{array}$ \\
\hline $\begin{array}{l}\text { Riqueza de las tareas matemáticas propuestas } \\
\text { o improvisadas en el transcurso de la } \\
\text { enseñanza (RT) }\end{array}$ & $\begin{array}{l}\text { Procesos cognitivos: Propone tareas matemáticas de } \\
\text { reproducción, conexión y reflexión durante la enseñanza. }\end{array}$ \\
$\begin{array}{l}\text { Situación: Propone tareas matemáticas de carácter personal, } \\
\text { educativo/profesional, público y científico durante la enseñanza. } \\
\text { Contexto: Propone tareas matemáticas auténticas ehipotéticas } \\
\text { durante la enseñanza. }\end{array}$ \\
\hline $\begin{array}{l}\text { Coherencia de las tareas con las finalidades } \\
\text { educativas (CT) }\end{array}$ & $\begin{array}{l}\text { Descripción tarea, concepto y habilidades. } \\
\text { Claridad del camino de aprendizaje (CA) }\end{array}$ \\
\hline $\begin{array}{l}\text { Identifica capacidades. Destaca posibles errores. } \\
\text { Ias a areas con los significados, y }\end{array}$ & $\begin{array}{l}\text { Las tareas muestran diversos significados. Se adecúan a las } \\
\text { situaciones y a los contextos. }\end{array}$ \\
\hline
\end{tabular}

La información obtenida se organizó en seis episodios de clases. Cada episodio corresponde a una sesión de grabación (estas sesiones pueden corresponder a una o dos lecciones de clase, de 40 minutos cada una; en la educación secundaria en Costa Rica). El análisis realizado se basa en las categorías descritas, a las que se les asignaron los códigos EC, VC, SS, VS, DR, RSR, LM, RER, APE, RRE, RT, CT, CA, TTSC para cada categoría del subdominio de conocimiento especializado como se indica en la tabla 1.

\section{Análisis del conocimiento especializado del contenido}

Este apartado describe el conocimiento especializado sobre los conceptos básicos de función que manifiesta un profesor de educación secundaria al enseñar este tema. La información se presenta a partir del estudio de los conceptos y las tareas de aprendizaje. Se analizaron seis episodios de clases, con base en las categorías establecidas para profundizar en el CEC. El reconocimiento de información vinculada a categoría se identifica con las abreviaturas correspondientes, por ejemplo, para la categoría sobre el grado en que las situaciones dan sentido al contenido matemático escolar se utiliza [SS] como se detalló en la tabla 1. 
La información se acompaña con fragmentos de algunas de las intervenciones grabadas durante las lecciones (episodios). Aquellas hechas por el profesor se identifican con $P$ y las que corresponden a sus estudiantes con $E_{i}$ (el subíndice se empleará para distinguir las intervenciones estudiantiles. Por ejemplo, las indicaciones $\mathrm{E}_{1}$ y $\mathrm{E}_{2}$ refieren a diferentes estudiantes que dialogan con el profesor. Si se escribe la letra E (sin subíndice) es porque la intervención la realiza un grupo de estudiantes de forma simultánea). Para clarificar lo sucedido en los seis episodios, como preámbulo se muestra una descripción de estos (una síntesis).

\section{Descripción de los episodios}

En el primer episodio el profesor presenta un problema (una tarea) sobre la tarifa de un taxi con el objetivo de introducir el concepto de función, luego solicita a sus estudiantes que, en grupos, lo resuelvan. Una persona de cada grupo debe pasar a la pizarra a mostrar el procedimiento y la solución encontrada.

En el segundo episodio el profesor retoma el problema de la clase anterior y utiliza un folleto (material didáctico impreso elaborado por el profesor) para exponer conceptos, ejemplos y ejercicios.

Durante el episodio siguiente el profesor introduce y define el concepto de función, esto a partir del concepto de relación visto en la clase anterior, posteriormente asigna al estudiantado la resolución de algunos ejercicios propuestos en el folleto y le pide a una parte pasar a la pizarra a escribir las respuestas de los ejercicios.

En el episodio cuarto el profesor presenta en la pizarra los conceptos implicados en los ejercicios que se han resuelto; luego los retoma haciendo uso del folleto. Dicta un problema a sus estudiantes y posteriormente hacen la revisión en la pizarra.

En el quinto episodio el profesor se guía con el folleto para ejemplificar el cálculo del ámbito de una función y el cálculo de preimágenes. En el último episodio el profesor, guiado por el folleto, expone algunas tareas seleccionadas (ejemplos) en la pizarra.

\section{Contenidos matemáticos expuestos: Conceptos, representaciones y contextos}

Haciendo una reconstrucción de las clases grabadas, en el Episodio [1] se presentó un problema sobre la tarifa de un taxi, empleado para introducir el concepto de función, que abarcaba los conceptos sobre variable dependiente, variable independiente y ecuación [SS]. Analizando el episodio, se identifica que el profesor explicó cómo resolver la pregunta del problema en que un estudiante aplicó el concepto de ecuación [EC]. El problema propuesto se encontraba dentro del contexto de interés para sus estudiantes y forma parte de los ejes transversales propuestos en el Plan de Estudios (resolución de problemas), pues se refería al cálculo de la tarifa de un taxi, según los kilómetros adicionales e introducía la identificación de los elementos de una función [VC].

El profesor utilizó para este problema la representación simbólica algebraica y numérica para introducir los conceptos de variable dependiente y variable independiente [DR, RSR, RER]. Según el análisis realizado, empleó un lenguaje matemático preciso [LM]. Además, mostró disposición para responder con claridad y precisión a las preguntas y dudas de sus estudiantes [RRE].

El profesor destacó la variedad de procedimientos y otorgó validez a los algoritmos no convencionales propuestos por estudiantes [CA]. Esto se evidencia en algunas de las discusiones entre docente y estudiantes, como la siguiente. 
P: Ahora bien, la pregunta es si dispongo de ocho mil colones ¿cuántos kilómetros adicionales podrían viajar en el taxi?

$\mathrm{E}_{1:}$ Por ahí otro compañero me dijo, que lo que hicieron fue ir probando con un valor multiplicando seiscientos hasta llegando un aproximado casi a los ocho mil colones. Pero por ejemplo el compañero acá $\mathrm{E}_{2}$ me decía que él lo que hizo fue ¿qué? Explíquelo $\mathrm{E}_{2}, \ldots$ usted disponía de ocho mil colones ¿qué fue lo que hizo?

$\mathrm{E}_{2}$ : Le reste los seiscientos cinco

P: O sea solo dispongo de ocho mil, quiero saber cuántos kilómetros adicionales puedo recorrer, entonces sería seiscientos cinco más seiscientos “equis” ¿qué es esto para ustedes chicos?

\section{E: Una ecuación}

P: Una pequeña ecuación ¿cómo resuelvo yo una ecuación? ¿Entonces tiene lógica lo que dice $\mathrm{E}_{2}$ ? A ocho mil le quito seiscientos cinco y lo que me da lo divido ¿entre?

En la figura 2 se muestra al profesor considerando los procedimientos (y soluciones) propuestos por los subgrupos de estudiantes (Grupo 1, Grupo 2, Grupo 3 y Grupo 4) para destacar los conceptos de variable dependiente y variable independiente. Se evidencian en la imagen la representación simbólica y sus tipos: algebraica y numérica.

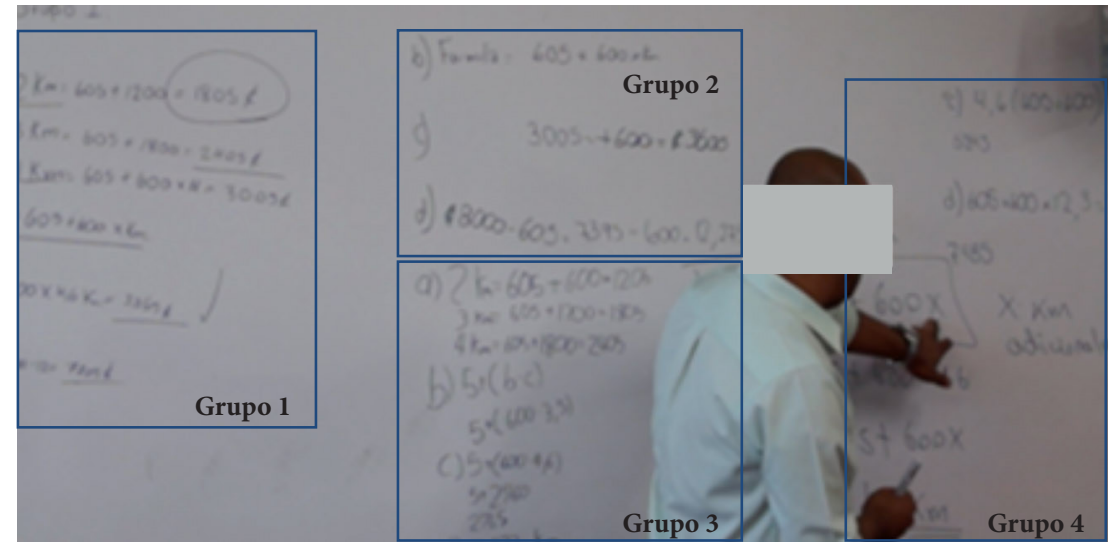

Figura 2. El profesor destaca los conceptos de variable dependiente e independiente. Tomada de las grabaciones de aula.

Durante el Episodio [2] el profesor retomó el problema descrito, empleando una sección de un folleto para exponer ejemplos, ejercicios y conceptos. Los temas abordados con la actividad fueron: cantidad constante, cantidad variable, variable dependiente, variable independiente, relación. Las explicaciones, ejemplos, ejercicios y problemas propuestos por el profesor mostraron la relación entre los conceptos enunciados [EC], como se observa en el siguiente fragmento. 
P: ... Un ejemplo diferente ya a la parte de física, dice, el peso esperado $\mathrm{P}$ en toneladas de una ballena adulta se relaciona con su longitud L, me dan una fórmula, por ahí el otro grupo me preguntaba, profesor usted siempre nos va a dar la formula, bueno vamos a ver que más adelante ustedes con algunos conocimientos que van a ir adquiriendo van a descubrir esa fórmula. Dice que el peso $\mathrm{P}$ en toneladas de una ballena adulta se relaciona su longitud $\mathrm{L}$ en metros mediante la ecuación peso es igual a cinco coma cincuenta y siete veces la longitud menos la constante cuarenta y dos coma ocho, esa es la fórmula, primera pregunta a) ¿cuál es la variable dependiente y cuál es la variable independiente?

E: P es la dependiente

P: Vamos con la variable dependiente en primera instancia, ¿quién sería?

E: P

P: Excelente, ¿variable independiente?

E: L

P: La longitud de la ballena en metros, el peso va a depender de la longitud que tenga la ballena, entonces es la variable independiente; también pudimos haber escrito así $\mathrm{P}$ en términos ¿de quién? De L, es exactamente lo mismo.

Asimismo, planteó situaciones para mostrar el uso de los conceptos de relación, cantidad constante, cantidad variable, variable dependiente y variable independiente [SS]. Para la mayoría de conceptos definidos en clase se mostró un único significado.

El docente utilizó una diversidad de sistemas de representación para los conceptos vistos en clase, por ejemplo, para el concepto de relación utilizó las representaciones icónicas, tabular, simbólica numérica y gráfica. Cabe destacar que el sistema de representación gráfico no se encuentra dentro del programa de estudio, sin embargo, esta representación es fundamental para adquirir el concepto de función, de variable dependiente e independiente [DR]. También se identificó que el profesor hizo un uso frecuente de los sistemas de representación verbal, simbólica algebraica y simbólica numérica. Para representar más de un concepto utilizó las representaciones verbal, tabular, simbólica algebraica y simbólica numérica [RSR]. Para el ejemplo del concepto de relación propuesto por el profesor, estableció una relación entre los sistemas de representación icónica y gráfico [RER]. En general, el profesor mostró riqueza, fluidez y precisión en el uso del lenguaje matemático [LM].

El Episodio [3] se caracterizó por la presentación de conceptos como función, dominio, codominio, ámbito, relación, diagrama, par ordenado y notación de una función. En general, durante las explicaciones y ejemplos el profesor manifestó en este episodio una riqueza en la relación de conceptos [EC].

P: No cumple ¿la palabra?

E: Único

P: Único, excelente, entonces ya no es una función, quiero que quede claro, es una relación y aquí podemos concluir algo importante, ¿toda función es?

E: Relación

P: Relación, pero no toda relación va a corresponder a una función, es algo que tienen que manejar siempre. 
UNICIENCIA Vol. 32, No. 1, pp. 89-107. Enero-Junio, 2018.

En este episodio el profesor plateó una tarea en un contexto de interés para sus estudiantes y la utilizó para identificar elementos de una función [VC]; asimismo esta tarea le permitió vincular conceptos como relación y función [SS], pero presentó un significado para cada uno de los conceptos [VS].

P: [...] las notas van desde un 0 hasta llegar a un ¿qué?

E: 100

P: Lo máximo que puede tener un estudiante es ¿un?

E: 100

P: Y lo mínimo que podría tener, que no haya hecho absolutamente nada, ¿es un? 0, y yo sé que aquí no se dio la situación. Supongamos que $\mathrm{E}_{1}$ obtuvo un 70 , todos hicieron el examen, vamos a excluir a $\mathrm{E}_{2}$ que fue el único que no me hizo el examen, pero todos los demás hicieron el examen, ok, entonces $\mathrm{E}_{1}$ obtuvo un 70 , ¿tiene lógica decir que $\mathrm{E}_{1}$ al mismo tiempo obtuvo un 100 ?

E: No

P: Carece de sentido lógico, ¿verdad?, es ahí donde dice la situación cada elemento tiene que tener un único elemento, una situación como esta es una función, ahora, ¿podría ser que $\mathrm{E}_{3}$, estoy inventando nombres, también obtenga un 70?

E: Sí

P: Por eso es que dos elementos diferentes si pueden ir a uno mismo, no hay problema con la relación para que sea una función.

Para el concepto de relación el profesor utilizó los sistemas de representación verbal, icónica, simbólica numérica y gráfico en un problema contextualizado a una situación personal; para el concepto de función utilizó estos mismos sistemas de representación, pero añadió el simbólico algebraico. También, introdujo los conceptos de dominio y codominio por medio de las representaciones verbal e icónica. El uso de la representación gráfica fue constante para explicar los conceptos [DR]. Los sistemas de representación más utilizados por el profesor fueron verbal, icónico, simbólico algebraico y simbólico numérico [RSR]; además estableció una relación entre el gráfico, icónico y simbólico numérico [RER]. El lenguaje matemático utilizado por el profesor fue fluido y preciso [LM].

En el inicio del Episodio [4] el profesor abordó, nuevamente, los contenidos sobre dominio, codominio, ámbito, preimagen, imagen y criterio de una función. Durante la enseñanza de estos conceptos mostró una riqueza en relaciones entre nociones [EC], como se observa en el fragmento siguiente.

P: Entonces todo par ordenado, vamos a tener ya dos nombres, preimagen-imagen, variable independiente-variable dependiente, o también podemos denominar “ $x$ "- “ $y$ ”, que más adelante que veamos el plano cartesiano, ya ubicar puntos en el plano cartesiano, siempre va a mantener este orden " $x$ " coma " $y$ ", primero la preimagen luego la imagen. Por ahí me preguntaba el compañero, que no entendía dos conceptos porque hablaba de formar un par ordenado donde hablaba de abscisa y ordenada, precisamente aquí la variable independiente, que es el valor de " $x$ ", es la que va a recibir el nombre de abscisa y la otra va a recibir el nombre de ordenada. 
El profesor presentó una tarea sobre "el vendedor de revistas", actividad que se encuentra dentro del contexto de aplicación y que se utiliza para identificar elementos de una función [VC]. Para esta situación introdujo el cálculo de preimagen e imagen [SS]. Además, presentó un significado para los conceptos dominio y criterio de una función; para definir preimagen, imagen, ámbito y codominio el profesor utilizó más de un significado [VS].

Durante la lección se mostró una diversidad de sistemas de representación para los conceptos enunciados. Por ejemplo, para el concepto dominio y codominio utilizó los modos: verbal, icónico, tabular, simbólico algebraico y simbólico numérico. El uso de las representaciones verbal, simbólica algebraica y simbólica numérica fue frecuente [DR, RSR] (figura 3). El docente estableció, por ejemplo, una relación entre las representaciones icónica, verbal, simbólica algebraica y simbólica numérica [RER].

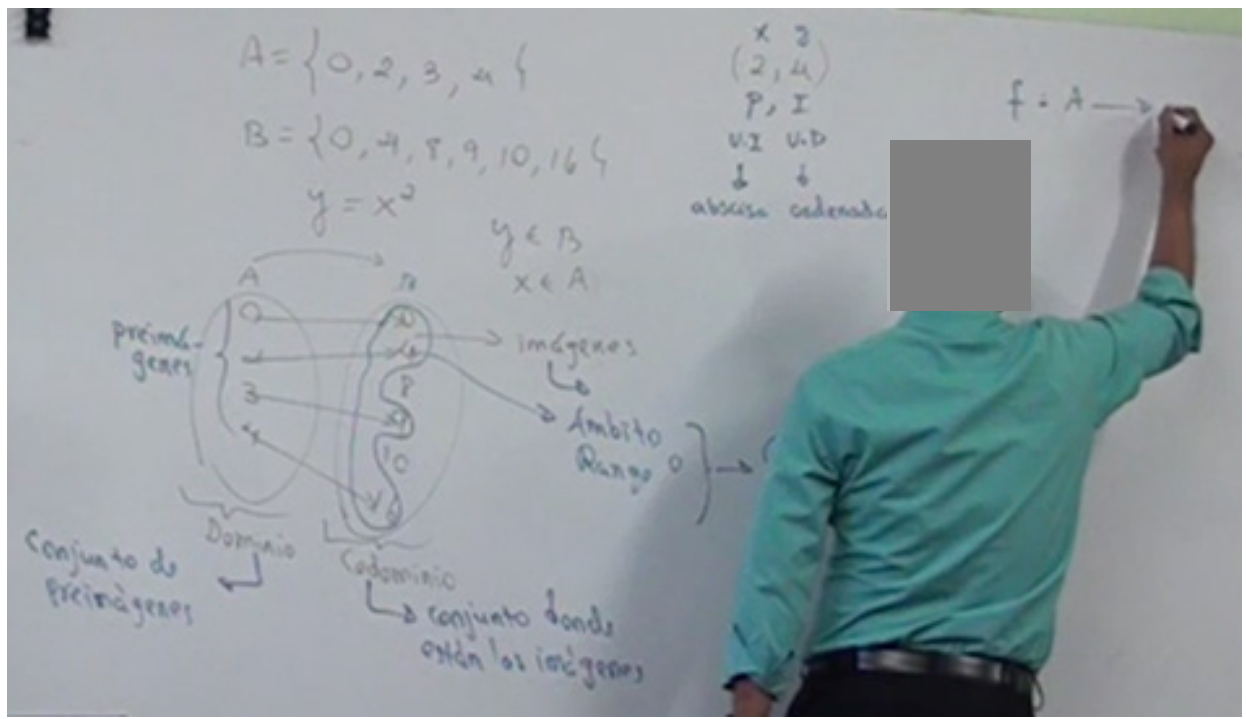

Figura 3. Uso de diversos tipos de sistemas de representación en el episodio 4. Tomada de las grabaciones de aula.

En el Episodio [5], al abordarse los contenidos de ámbito y preimagen, se observó una riqueza en la relación de conceptos. Por ejemplo, el profesor expuso una relación entre los conceptos dominio, codominio, ámbito, criterio de una función, imagen y el procedimiento de "sustitución" durante uno de los ejemplos planteados [EC].

Para los conceptos preimagen e imagen utilizó los sistemas de representación verbal, simbólica algebraica y numérica, pero para el concepto de preimagen usó, además, la icónica en uno de los ejemplos presentados [DR]. Los sistemas de representación verbal, simbólica algebraica y simbólica numérica fueron de uso frecuente y se mostraron para más de un concepto [RSR]. El profesor, en uno de los ejemplos, utilizó el sistema de representación icónica y simbólica numérica estableciendo una relación entre estos [RER]. Incluyó el sistema de representación tabular cuando trabajaba el concepto de codominio [DR, RSR], y estableció relaciones entre representaciones [RER].

Finalmente, en el Episodio [6] el profesor, en sus explicaciones, relacionó los conceptos de gráfica, gráfico y pares ordenados [REC]. Para el concepto de gráfica presentó solo un significado [VS]. Para los conceptos función, imagen y preimagen utilizó los sistemas de representación 
tabular, gráfica y gráfico [DSR]. En los ejemplos propuestos por el profesor se mostró una relación entre los sistemas de representación tabular y gráfica [ER]. El lenguaje matemático utilizado por el docente fue rico, fluido y preciso [APL].

La tabla 2 presenta una síntesis de los conceptos, sistemas de representación y contextos identificados en los episodios analizados.

Tabla 2

Conceptos, sistemas de representación y contextos

\begin{tabular}{|c|c|c|c|c|c|}
\hline Episodio 1 & Episodio 2 & Episodio 3 & Episodio 4 & Episodio 5 & Episodio 6 \\
\hline \multicolumn{6}{|c|}{ Conceptos } \\
\hline $\begin{array}{l}\text { Variable, variable } \\
\text { dependiente e } \\
\text { independiente, } \\
\text { ecuación }\end{array}$ & $\begin{array}{l}\text { Cantidad } \\
\text { constante, } \\
\text { cantidad } \\
\text { variable, variable } \\
\text { dependiente e } \\
\text { independiente, } \\
\text { relación }\end{array}$ & $\begin{array}{l}\text { Función, } \\
\text { dominio, } \\
\text { codominio, } \\
\text { ámbito, relación, } \\
\text { diagrama, par } \\
\text { ordenado }\end{array}$ & $\begin{array}{l}\text { Dominio, } \\
\text { codominio, } \\
\text { ámbito, } \\
\text { preimagen, } \\
\text { imagen, criterio } \\
\text { de una función. }\end{array}$ & $\begin{array}{l}\text { Dominio, } \\
\text { codominio, } \\
\text { ámbito, } \\
\text { preimagen, } \\
\text { imagen, criterio }\end{array}$ & $\begin{array}{l}\text { Función, gráfico, } \\
\text { gráfica, par } \\
\text { ordenado }\end{array}$ \\
\hline \multicolumn{6}{|c|}{ Sistemas de representaciones } \\
\hline $\mathrm{V}, \mathrm{SN}, \mathrm{SA}$ & $\mathrm{V}, \mathrm{I}, \mathrm{T}, \mathrm{G}, \mathrm{SN}, \mathrm{SA}$ & $\mathrm{V}, \mathrm{I}, \mathrm{G}, \mathrm{SN}, \mathrm{SA}$ & $\mathrm{V}, \mathrm{T}, \mathrm{I}, \mathrm{SN}, \mathrm{SA}$ & $\mathrm{V}, \mathrm{I}, \mathrm{SN}, \mathrm{SA}$ & $\mathrm{V}, \mathrm{T}, \mathrm{G}, \mathrm{SN}, \mathrm{SA}$ \\
\hline \multicolumn{6}{|c|}{ Contextos } \\
\hline $\begin{array}{l}\text { Matemático } \\
\text { (cálculo) } \\
\text { Social (transporte } \\
\text { público) }\end{array}$ & $\begin{array}{l}\text { Científico } \\
\text { matemático } \\
\text { (cálculo) }\end{array}$ & Matemático & $\begin{array}{l}\text { Cálculo } \\
\text { matemático } \\
\text { Comercial }\end{array}$ & $\begin{array}{l}\text { Cálculo } \\
\text { matemático } \\
\text { Educativo }\end{array}$ & $\begin{array}{l}\text { Cálculo } \\
\text { matemático }\end{array}$ \\
\hline
\end{tabular}

Nota. V: Verbal; I: icónico; T: tabular; G: gráfico; SN: simbólico numérico; SA: simbólico algebraico

\section{Tareas de aprendizaje}

La tarea central del Episodio [1] fue el problema propuesto sobre la tarifa del taxi para introducir el tema de funciones. Esta constituyó una tarea de reflexión, de carácter público y auténtica. Además, el problema respondía a una habilidad específica del programa de estudio: "distinguir cantidades constantes y variables" (MEP, 2013, p. 14); además, se adecua a los contextos y situaciones [RT, CT, TSSC].

En el Episodio [2] el profesor planteó tareas que hacían referencia a contextos de interés para los estudiantes (como el científico), de aplicación y sobre historia de la matemática. Estas fueron utilizadas para identificar elementos de una función [VC]. Por ejemplo, destacó "Según las últimas investigaciones médicas, después de consumir unos dos paquetes completos de cigarrillos, por cada paquete adicional de cigarrillos que consume un ser humano, se daña aproximadamente 3 bronquios de sus pulmones. De acuerdo a la información anterior podríamos obtener la siguiente ecuación , $B=3 p-6$ donde $B$ es la cantidad de bronquios afectados después de consumir $p$ paquetes de cigarrillos". 
Durante el Episodio [3] se propusieron tareas de reproducción y conexión. Se trataron situaciones de carácter personal, educativo y científico (crecimiento, relación de áreas). Las tareas propuestas se clasifican como hipotéticas y auténticas [RT]; estas mostraron diversos significados de algunos conceptos y se adecuaban a las situaciones y contextos indicados [TSSC].

El cuarto episodio se caracterizó por la presentación de tareas de reproducción, por ejemplo, identificar por medio de diagramas relaciones que corresponden a funciones. Las tareas eran de carácter educativo, hipotéticas y auténticas [RT]. Además, todas las tareas correspondían a la habilidad de identificar relaciones entre una función [CT].

En el Episodio [5] las tareas propuestas, en su mayoría, fueron de reproducción; sin embargo, los estudiantes, en una de las tareas, debían encontrar una fórmula para una función estableciendo conexiones matemáticas que conducían a la generalización. La mayor parte de las tareas fueron de carácter educativo con la excepción del problema del vendedor de revistas que puede catalogarse de carácter público. La mayoría de las tareas desarrolladas durante la clase fueron hipotéticas [RT]. Estas respondían a la habilidad del programa de estudio sobre la identificación del dominio, codominio, ámbito, imágenes y preimágenes de una función [CT]. Las tareas planteadas por el profesor mostraron una variedad de significados, se adecuaban a diversas situaciones y contextos [TSSC].

Por último, en el Episodio [6] las tareas planteadas por el docente eran de reproducción, sobre cálculo del ámbito de una función y de alguna preimagen específica. Estas tareas desarrolladas en la clase eran de carácter educativo e hipotético [RT] y respondían a las habilidades de identificar el dominio, codominio, ámbito, imágenes y preimágenes de una función, y evaluar el valor de una función representada gráficamente a partir de distintos puntos de su dominio [CT]. La tabla 3 destaca algunas de las particularidades de las tareas propuestas en los seis episodios analizados.

Tabla 3

Caracterización de las tareas propuestas en los episodios

\begin{tabular}{|c|c|c|c|c|c|}
\hline Episodio 1 & Episodio 2 & Episodio 3 & Episodio 4 & Episodio 5 & Episodio 6 \\
\hline \multicolumn{6}{|c|}{ Tareas } \\
\hline $\begin{array}{l}1 \text { tarea: } \\
\text { Reflexión } \\
\text { Auténtica } \\
\text { Responde a } \\
\text { una habilidad } \\
\text { específica } \\
\text { (identificar) }\end{array}$ & $\begin{array}{l}\text { Más de } 1 \text { tarea } \\
\text { Reproducción y } \\
\text { conexión } \\
\text { Hipotéticas y } \\
\text { auténticas } \\
\text { Dos habilidades } \\
\text { específicas } \\
\text { (identificar y } \\
\text { aplicar) }\end{array}$ & $\begin{array}{l}\text { Más de } 1 \text { tarea } \\
\text { Reproducción } \\
\text { Hipotéticas y } \\
\text { auténticas } \\
\text { Responden a } \\
\text { una habilidad } \\
\text { (identificar) }\end{array}$ & $\begin{array}{l}\text { Más de } 1 \text { tarea } \\
\text { Reproducción, } \\
\text { conexión } \\
\text { Hipotéticas } \\
\text { Responden a } \\
\text { una habilidad } \\
\text { (identificar) }\end{array}$ & $\begin{array}{l}\text { Más de } 1 \text { tarea } \\
\text { Reproducción } \\
\text { Hipotéticas } \\
\text { Responden a } \\
\text { una habilidad } \\
\text { (identificar) }\end{array}$ & $\begin{array}{l}\text { Más de } 1 \text { tarea } \\
\text { Reproducción } \\
\text { Hipotéticas } \\
\text { Responden } \\
\text { a varias } \\
\text { habilidades } \\
\text { (trazar, } \\
\text { calcular) }\end{array}$ \\
\hline
\end{tabular}

En general, el profesor presenta una variedad de tareas durante la enseñanza de los conceptos básicos de función, sin embargo, sobresale el uso de situaciones de reproducción y conexión en contextos educativos y que responden principalmente a la habilidad de identificar.

Junto a esta descripción tabular sobre los conceptos matemáticos abordados y las tareas de aprendizaje propuestas, en la tabla 4 se destacan las categorías de análisis sobresalientes. 
UNICIENCIA Vol. 32, No. 1, pp. 89-107. Enero-Junio, 2018.

Email: revistauniciencia@una.cr

Tabla 4

Reconocimiento de categorías de análisis por episodio

\begin{tabular}{|c|c|c|c|c|c|c|c|c|c|c|c|c|}
\hline \multirow[t]{2}{*}{$\mathrm{E}$} & \multicolumn{12}{|c|}{ CATEGORÍA } \\
\hline & $\mathrm{EC}$ & $\mathrm{VC}$ & SS & VS & $\mathrm{DR}$ & RSR & LM & RER & RRE & RT & CT & TSSC \\
\hline 1 & 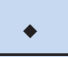 & 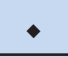 & 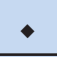 & & - & - & - & - & 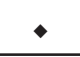 & - & - & - \\
\hline 2 & - & - & 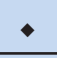 & & - & 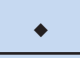 & 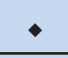 & - & & & & \\
\hline 3 & 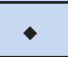 & 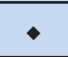 & 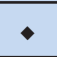 & 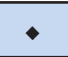 & - & - & 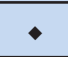 & 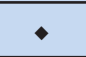 & & 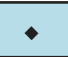 & & - \\
\hline 4 & 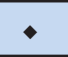 & - & 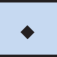 & - & - & 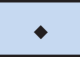 & & & & 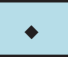 & - & \\
\hline 5 & 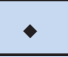 & & & & - & - & & 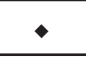 & & 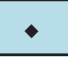 & 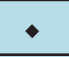 & - \\
\hline 6 & . & & & 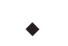 & & • & • & - & & • & • & \\
\hline
\end{tabular}

Nota. - Se observa E: episodio; EC: Riqueza estructura conceptual; VC: variedad de contextos; SS: situaciones que dan sentido; VS: variedad de significados; DR: diversidad de sistemas de representación; RSR: realce a sistemas de representación; LM: lenguaje matemático; RER: relaciones entre representaciones; RRE: riqueza de respuestas; RT: riqueza de tareas; CT: coherencia de tareas; CA: claridad del camino de aprendizaje; TSSC: Relación tareas, significados y contextos.

La distribución de datos mostrada en la tabla 4 destaca la presencia de aspectos sobre la riqueza de la estructura conceptual y los sistemas de representación a lo largo de los seis episodios. Asimismo, se aprecia en los primeros tres episodios un énfasis, que es constante, en la variedad de contextos, las situaciones que dan sentido a estos, el uso de una variedad de representaciones y las relaciones entre estas, y la calidad del lenguaje matemático mostrado en estos momentos.

Los datos obtenidos acentúan las unidades de análisis sobre presentación de tareas, en los episodios finales del desarrollo del tema. En los episodios 4, 5 y 6 se aprecia una riqueza en la presentación de tareas y destaca su coherencia con el tema. El episodio 1 sobresale por la presencia de la gran mayoría de aspectos considerados para el análisis (solo es excepción la categoría de variedad de significados).

De acuerdo con el análisis de los episodios de clases, en el apartado siguiente se establecen algunas conclusiones sobre el conocimiento especializado del profesor participante en el estudio, en cuanto a la enseñanza de los conceptos básicos de función.

\section{Consideraciones finales}

A pesar de la implementación de las recientes directrices curriculares del Ministerio de Educación Pública de Costa Rica, en cuanto a la metodología de resolución de problemas como principal estrategia de enseñanza, en general la dinámica de clase que sigue el profesor del estudio se reduce a la presentación de conceptos matemáticos, seguida por la exposición y asignación de una serie de tareas que el grupo de estudiantes resuelve y presenta sus resultados en la pizarra donde son validadas por el docente. Excepto en el primer episodio en que introduce el concepto de relación mediante la estrategia de resolución de problemas.

Con especificidad, se destaca la riqueza de la estructura conceptual presentada durante la enseñanza de los conceptos básicos de función. Sobresale también el uso frecuente de distintos sistemas de representación y la relación entre ellas en los primeros episodios del desarrollo del tema. Incluso con frecuencia utiliza una misma representación para mostrar más de un concepto, como sucedió con los conceptos de par ordenado, imagen y preimagen en el que empleó la representación simbólica. 
ISSN Electrónico: 2215-3470

DOI: http://dx.doi.org/10.15359/ru.32-1.6
UNICIENCIA Vol. 32, No. 1, pp. 89-107. Enero-Junio, 2018. URL: www.revistas.una.ac.cr/uniciencia Email: revistauniciencia@una.cr

Se concluye que la presentación de los conceptos matemáticos (vinculados o no al tema de funciones) disminuye en los episodios finales, cuando resalta la presentación y asignación de tareas y aparecen con mayor frecuencia la variedad de contextos.

Por su parte, existe ausencia de una riqueza en las respuestas proporcionadas por el profesor a lo largo de los episodios. Cabe mencionar que, a pesar de que se identifican respuestas adecuadas, la mayoría de estas no responden a una variedad según las categorías de análisis consideradas.

Otra consideración destacable es el uso adecuado y preciso del lenguaje matemático por parte del profesor durante el desarrollo del tema, tanto al momento de exponer los conceptos como al responder dudas y preguntas de sus estudiantes.

En relación con las tareas presentadas por el docente, se caracterizan principalmente por proponer más de una situación en cada episodio, excepto en el primero. La tarea propuesta en este episodio sobresale entre las demás por tener un grado de complejidad mayor y por ser empleada para introducir un concepto que no había sido analizado en clase. Las demás tareas se presentan principalmente para reforzar conocimientos y para mostrar distintos contextos en que pueden movilizarse algunos de los conceptos básicos de función, las que prevalecen corresponden a tareas de reproducción. En general, el contexto más empleado es el hipotético y en su mayoría responden a una de las habilidades propuesta en el Plan de Estudios (MEP, 2012).

En general, la metodología de enseñanza del profesor es tradicional; sin embargo, muestra un alto conocimiento especializado del tema de función. Su desempeño en el aula -durante las lecciones observadas- destaca también habilidades para la docencia, pesar de la implementación evidente de las directrices curriculares sobre la resolución de problemas matemáticos. Desde una óptica general se percibe cómo la presentación de conceptos sigue predominando al inicio de la clase, seguido de un énfasis en la presentación, asignación y resolución de tareas vinculadas al tema, que no sobrepasan a niveles de conexión y reflexión.

\section{Reconocimientos}

Este artículo es producto del Proyecto SIA 0127-13 titulado Conocimiento matemático para la enseñanza de los profesores sobre el tema de funciones en el Ciclo Diversificado de la Educación Matemática en Costa Rica que se desarrolla en la Escuela de Matemática de la Universidad Nacional de Costa Rica.

\section{Referencias}

Agarwal, S. (2006). The Nature of Pre-service Secondary Mathematics Teachers' Knowledge of Mathematics for Teaching of Functions (Tesis doctoral). University of New York At Buffalo, Estados Unidos de América.

Ball, D., Hill, H. y Bass, H. (2005). Knowing mathematics for teaching: Who knows mathematics well enough to teach third grade, and how can we decide? American Educator, 29(1), 14-17, 20-22, 43-46. Recuperado de http://hdl.handle.net/2027.42/65072

Ball, D., Thames, M. y Phelps, G. (2008). Content Knowledge for Teaching: What Makes It Special? Journal of TeacherEducation, 59(5), 389-407.doi:https://doi.org/10.1177/0022487108324554

Clement, L. (2001). What do students really know about functions? Mathematics Teacher of The National Council of Teachers of Mathematics, 94(9), 745-748. Disponible en $\quad$ http://www.nctm.org/Publications/mathematics-teacher/2001/Vol94/Issue9/ Connecting-Research-to-Teaching -What-Do-Students-Really-Know-about-Functions / 
UNICIENCIA Vol. 32, No. 1, pp. 89-107. Enero-Junio, 2018.

ISSN Electrónico: 2215-3470

URL: www.revistas.una.ac.cr/uniciencia

DOI: http://dx.doi.org/10.15359/ru.32-1.6

Climent, N. (2002). El desarrollo profesional del maestro de primaria respecto de la enseñanza de la matemática. Un estudio de caso (Tesis doctoral). Universidad de Huelva, España.

Espinoza, J., Espinoza, J., González, M., Ramírez, I. y Zumbado, M. (2008). La resolución de problemas en la enseñanza de las matemáticas: Una experiencia con la función exponencial, polígonos y estadística (Memoria de seminario de licenciatura). Universidad Nacional de Costa Rica, Heredia, Costa Rica.

Gómez, P. (2007). Desarrollo del conocimiento didáctico en un plan de formación inicial de profesores de matemáticas de secundaria (Tesis doctoral). Universidad de Granada, España.

Haciomeroglu, G. (2006). Prospective secondary teachers' subject matter knowledge and pedagogical content knowledge of the concept of function (Tesis doctoral). The Florida State University College of Education, Estados Unidos de América.

Hill, H., Ball, D. y Schilling, S. (2008). Unpacking Pedagogical Content Knowledge: Conceptualizing and Measuring Teacher's Topic-Specific Knowledge of Students. Journal for Research in Mathematics Education, 39(4), 372-400. Recuperado de http://www. nctm.org/Publications/journal-for-research-in-mathematics-education/2008/Vol39/ Issue4/Unpacking-Pedagogical-Content-Knowledge -Conceptualizing-and-MeasuringTeachers -Topic-Specific-Knowledge-of-Students/

Lupiáñez, J. (2009). Expectativas de aprendizaje y planificación de formación inicial de profesores de matemáticas de secundaria (Tesis doctoral). Universidad de Granada, España.

McMillan, J. y Schumacher, S. (2005). Investigación educativa (5ª ed). Madrid, España: Pearson Educación.

Ministerio de Educación Pública. (2012a). Informe nacional 2011. Resultados de las pruebas nacionales de bachillerato de la educación formal. San José, Costa Rica: Dirección de Gestión y Evaluación de la Calidad.

Ministerio de Educación Pública. (2012b). Programa de Estudios de Matemáticas. Educación General Básica y Ciclo Diversificado. San José, Costa Rica: Autor. Recuperado de http:// www.reformamatematica.net/comunidaded/sites/default/files/programas.pdf

Ministerio de Educación Pública. (2013). Programas de Estudio en Matemáticas, Transición2014. Recuperado de http://www.mep.go.cr/sites/default/files/programadeestudio/programas/ matematicatransicion2014.pdf

Morales, Y. y Font, V. (2017). Análisis de la reflexión presente en las crónicas de estudiantes en formación inicial en educación matemática durante su periodo de práctica profesional. Revista ACTA SCIENTIAE, 19(1), 122-137. Descargado de http://www.periodicos.ulbra.br/ index.php/acta/article/view/2975/2280

Morales-López, Y. (2017). Costa Rica: The Preparation of Mathematics Teachers. In A. Ruiz (Ed.), Mathematics Teacher Preparation in Central America and the Caribbean (pp. 39-56). Springer International Publishing. doi http://dx.doi.org/10.1007/978-3-319-44177-1 3

National Council of Teachers of Mathematics [NCTM]. (2000). Principles and Standards for School Mathematics. Reston, VA: Autor.

Programa Estado de la Nación en Desarrollo Humano Sostenible. (2013). Cuarto Informe Estado de la Educación (4 $4^{\text {ta }}$ ed.). San José, Costa Rica: Editorama. 
ISSN Electrónico: 2215-3470

DOI: http://dx.doi.org/10.15359/ru.32-1.6
UNICIENCIA Vol. 32, No. 1, pp. 89-107. Enero-Junio, 2018. URL: www.revistas.una.ac.cr/uniciencia Email: revistauniciencia@una.cr

Rodríguez-Flores, A., Picado-Alfaro, M., Espinoza-González, J., Rojas-González, N. y FloresMartínez, P. (2016). Conocimiento común del contenido que manifiesta un profesor al enseñar los conceptos básicos de funciones: Un estudio de caso. UNICIENCIA, 30(1), 1-16. doi: http://dx.doi.org/10.15359/ru.30-1.1

Rojas, N. (2010). Conocimiento para la enseñanza y calidad matemática de la instrucción del concepto de fracción: Estudio de caso de un profesor chileno. Memoria de trabajo de investigación tutelada no publicada. Granada, España: Departamento de Didáctica de la Matemática de la Universidad de Granada, España. http://fqm193.ugr.es/media/grupos/ FQM193/cms/Nielka Rojas.pdf

Rojas, N., Carrillo, J. y Flores, P. (2012). Características para identificar a profesores de matemáticas expertos. En A. Estepa, Á. Contreras, J. Deulofeu, M. Penalva, F. García y L. Ordoñez (Eds.), Investigación en Educación Matemática XVII (pp. 479-485), Jaén, España: SEIEM.

Rojas, N., Flores, P. y Ramos, E. (2013). El análisis didáctico como herramienta para identificar conocimiento matemático para la enseñanza en la práctica. En L. Rico, J. L. Lupiáñez, y M. Molina, Análisis didáctico en educación matemática. Metodología de investigación, formación de profesores e innovación curricular (pp. 191-208). Granada, España: Comares.

Ruiz, A., Barrantes, H. y Gamboa, R. (2009). Encrucijada en la enseñanza de la matemática: La formación de educadores. Cartago, Costa Rica: Editorial Tecnológica de Costa Rica.

Shulman, L. (1986). Those Who Understand: Knowledge growth in Teaching. Educational Researcher, 15(2), 4-14. doi: https://doi.org/10.3102/0013189X015002004

Shulman, L. (1987). Knowledge and Teaching: foundations of the New Reform. Harvard Educational Review, 57(1), 1-22. doi: https://doi.org/10.17763/haer.57.1.j463w79r56455411

Shumway, M. (2003). Preservice mathematics teachers' knowledge of functions and its effect on lesson planning at the secondary level (Tesis doctoral). The University of Iowa, Estados Unidos de América.

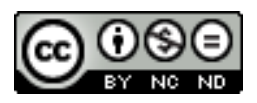

El conocimiento especializado del profesor de matemáticas: un estudio de caso sobre la enseñanza de los conceptos básicos de función (Ariana Rodríguez-Flores y otros) por Revista Uniciencia se encuentra bajo una Licencia CreativeCommons Atribución-NoComercial-SinDerivadas 3.0 Unported. 